\title{
Laparoscopic ureteroureterostomy for treatment of retrocaval ureter
}

\author{
Mario Álvarez-Maestro ${ }^{1,2,3}$, Diego M. Carrion,1,2 , Juan Gómez Rivas ${ }^{1,2,3}$, Jose Antonio Moreno Valle ${ }^{1,2,3}$, \\ Jose Quesada-Olarte ${ }^{1,2}$, Alfredo Aguilera Bazan ${ }^{1,2,3}$, Luis Martínez-Piñeiro ${ }^{1,2,3}$ \\ ${ }^{1}$ La Paz University Hospital, Department of Urology, Madrid, Spain \\ ${ }^{2}$ Universidad Autonoma de Madrid, Madrid, Spain \\ ${ }^{3}$ La Paz University Hospital Institute for Health Research (IdiPAZ), Madrid, Spain
}

Article history

Submitted: Nov. 25, 2018

Accepted: Dec. 26, 2018

Published online: Dec. 27, 2018

Citation: Álvarez-Maestro M, Carrion DM, Gómez Rivas J, et al. Laparoscopic ureteroureterostomy for treatment of retrocaval ureter. Cent European J Urol. 2019; 72: 75

\section{Key Words: laparoscopy () ureteroureterostomy (s retrocaval ureter \\ () minimally invasive surgery () ureteral surgery}

Retrocaval or circumcaval ureter is a rare congenital anomaly due to an anomalous development of the inferior vena cava (approximate incidence of one in 1000 live births), three to four times more common in males and with a predominance for the right side. The classification of Bateson and Atkinson differentiates two types: type I has an 'S' or 'fishhook' appearance and usually presents with symptomatic obstruction of the affected urinary tract, and type II, which has a less angulated 'sickle-shaped' ureteral deformity. Since the first reported surgical treatment for this condition, described by Anderson and Hynes in 1949 [Anderson JC, Hynes W. Retrocaval ureter; a rare case diagnosed pre-operatively and treated successfully by a plastic operation. Br J Urol. 1949; 21: 109-114] minimally invasive surgical approaches have gained popularity in the past several years. In the following video, we present our technique for transperitoneal laparoscopic ureteroureterostomy of a right retrocaval ureter without excision of the retrocaval segment. We present the case of a 38-year-old male, who was seen in the emergency department for right flank pain with associated acute renal failure. An abdominal ultrasound performed in an acute setting described findings compatible with that of right ureteropelvic junction obstruction. The patient underwent a right double
$\mathrm{J}$ stent placement as an emergency procedure and was discharged the following day with improvement in symptoms and laboratory analysis (serum creatinine and eGFR). As part of the outpatient evaluation, a computed tomography scan was performed, showing a right retrocaval ureter. A laparoscopic transperitoneal approach was chosen for the elective surgical repair.

In the left lateral decubitus position, a camera trocar, and 3 working trocars were positioned. Our surgical technique is described in a step-by-step manner in the video. The patient recovered well from surgery and was discharged home 48 hours later. The double $\mathrm{J}$ stent (placed during surgery) was removed 6 weeks after, and right side distal urine passage was seen in intravenous pyelography. An improvement was also noted in renal scintigraphy and diuretic renogram. The repair of a right retrocaval ureter with a laparoscopic transperitoneal ureteroureterostomy seems to be a feasible and safe approach with good results.

\section{CONFLICTS OF INTEREST}

The authors declare no conflicts of interest.

\footnotetext{
Corresponding author

Diego M. Carrion

diegocarrionm@hotmail.com
} 